scheinung. Es ist übrigens nicht schwer, unter den Symptomen, welche frische Apoplexieen darbieten, solche aufzufinden, welche gar nicht anders erklärt werden können, als durch eine Hemmung auf dem Nervenwege. Wenn z. B. angegeben wird, dass bei einem Apoplektiker, der einen Bluterguss im linken Grosshirn erlitten hat, das Spiel der Athemmuskeln rechterseits für Zeit geschwächt wird, so kann ja in diesem Fall unmöglich des Centrum der Athmung direkt geschädigt sein, da es so fern von dem Bluterguss, nämlich im verlängerten Mark seinen Sitz hat. Dieses Centrum kann also nur auf dem Nervenwege für Zeit gehemmt sein. Noch klarer liegt die Sache bei einer Lähmung der Blase, wie sie ja auch bei Apoplexien im Gehirn beobachtet werden kann. Das Centrum für die reflektorische Blasenentleerung liegt, wie ich bewiesen habe, tief unten im Lendenmark. Wird dieses Centrum in Folge einer Blutergiessung im Gehirn für Zeit gelähmt, so muss also eine Hemmung vorliegen, deren Nervenbahn innerhalb des Rückenmarks zu suchen ist, da das Gehirn nur durch Vermittelung des Rückenmarks diesen hemmenden Einfluss auf das Blasencentrum ausüben konnte.

Ich verzichte darauf, auf die zahlreichen anderen Anologieen, die sich zwischen den Erscheinungen nach der Gehirndurchspülung und den Folgen einer Hämorrhagie des Gehirns darbieten, noch besonders hinzuweisen, da diese von selbst einleuchten.

(Aus dem physiolog. Institute zu Innsbruck.)

\title{
Nervus Glossopharyngeus und Schmeckbecher.
}

Beobachtungen vou

\section{M. v. Vintschgau und J. Hönigschmied.}

Allen Physiologen wohl bekannte Versuche haben mit voller Sicherheit den Nachweis geliefert, dass der N. glossopharyngeus der Geschmacksnerv für den hinteren Theil der Zunge sei und es werden die von Lovén und $\mathrm{Schwalbe}$ gleichzeitig entdeckten Schmeckbecher von den meisten Physiologen als die Endorgane des 
genannten Nerven angesehen. Um jedoch diese Ansicht zu einer feststehenden Thatsache umzuwandeln, ist es aber unumgänglich nothwendig, den Zusammenhang des Glossopharyngeus mit den Schmeckbechern sicher nachweisen zu können. Man hat sich wohl bemüht, diesen Nachweis auf mikroskopischem Wege zu liefern, doch sind die bisher erhaltenen Resultate noch immer sehr spärlich.

Wenn man die Literatur über diesen Gegenstand näher prüft, so wird man wohl recht bald finden, dass blos Hönigschmied ${ }^{1}$ ) und kurze Zeit darauf Sertoli ${ }^{2}$ ) einige positive Angaben über jenen vermutheten Zusammenhang veröffentlicht haben. Die Beschreibung und die Abbildung der Art und Weise, wie die feinen Nervenfasern zu den Schmeckbechern gelangen, sind jedoch bei beiden Forschern etwas verschieden. Diesem Umstande ist es wahrscheinlich zuzuschreiben, dass auch in den neuesten Lehrbüchern der Physiologie diese beiden Beobachtungen nicht erwähnt werden und was noch wichtiger ist, dass die Verbindung der Schmeckbecher mit Nervenfasern als blos wahrscheinlich hingestellt wird.

Wir haben uns desshalb die Aufgabe gestellt, diese Frage vor der Hand wo möglich auf experimentellem Wege zu entscheiden und sind von der Idee ausgegangen, dass, wenn die Schmeckbecher wirklich Endorgane von Nervenfasern sind, dieselben eine Veränderung erleiden müssen, sobald man den entsprechenden Nerv durchschneidet. Der einzige in diesem Falle in Betracht kommende Nerv war der Glossopharyngeus.

Seine Durchschneidung geschah in bekannter Weise $^{3}$ ) und wir exstirpirten jedesmal 5 bis $8 \mathrm{Mm}$. des Nerven.

Am 6. Juni 1876 durchschnitten wir den rechten Glossopharyngeus an einem Kaninchen; die Zunge desselben wurde am 13. November näher untersucht (Kaninchen Nr. 1). Am 27. Juni 1876 durchschnitten wir den linken Glossopharyngeus an einem weissen Kaninchen, dessen Zunge am 18. November 1876 der Untersuchung unterzogen wurde (Kaninchen Nr. 2).

1) Beiträge zur mikroskopischen Anatomie über die Geschmacksorgane der Säugethiere. Zeitschrift für wissensch. Zoologie. XXIII. Band S. 414.

2) Osservazioni sulle terminazioni dei nervi del gusto. Gazzetta MedicoVeterinaria anno IV. 2 und auch Moleschott's Untersuch. z. Naturlebre XI. 4. Heft.

3) Frause, Anatomie des Kaninchens. 1868, und Cyon Methodik der physiologischen Experimente und Vivisectionen. 1874. 
Beide Kaninchen waren zur Zeit als wir die Operation vornahmen halberwachsen, und es wuchsen dieselben in den nachher verstrichenen fünf Monaten $\mathrm{zu}$ stattlichen Thieren heran.

Die Kaninchen wurden durch Verletzung des Herzens rasch getödtet, die Zunge alsogleich herausgeschnitten.

Die makroskopische Untersuchung zeigte keine Verschiedenheit in den beiden Zungenhälften. Nun wurden die Papillae foliatae und circumvallatae auf beiden Seiten herausgeschnitten und in eine $1 \%$ Ueberosmiumsäurelösung gegeben, in welcher dieselben zwischen 15-20 Stunden blieben. Es ist selbstverständlich, dass, um Irrthümer zu vermeiden, die rechte Papilla foliata und circumvallata getrennt von der linken aufbewahrt wurde.

Die anatomische Untersuchung ergab, dass an beiden Kaninchen die Operation richtig ausgeführt wurde, und dass keine Wicdervereinigung der Nervenstümpfe stattfand.

Die mikroskopische Untersuchung wies nach, dass bei beiden Kaninchen auf der gesunden Seite (Glossopharyngeus unversehrt) die Schmeckbecher, sowohl in der Pap. foliata als auch in der circumvallata, vollkommen unverändert waren; auf der operirten Seite (Glossopharyngeus durchschnitten) waren dagegen die Schmeckbecher verschwunden.

Ueber diesen Befund müssen wir etwas länger sprechen.

In den zahlreichen Präparaten aus der Pap. foliata des II. Kaninchens war es (mit Ausnahme eines einzigen) uns nicht möglich, die geringste Spur von Schmeckbechern oder deren Ueberbleibsel zu entdecken. Sowohl die Zellen, die man als Deckzellen bezeichnet, als auch jene, welche Geschmackszellen genannt werden, waren vollkommen verschwunden, an ihrer Stelle fanden wir blos gewöhnliche Epithelzellen, die so angeordnet waren, dass nicht die leiseste Spur zurïckblieb, aus welcher zu entnehmen gewesen wäre, es seien je in jener Gegend Geschmacksknospen vorgekommen.

In dem oben erwähnten Präparate fanden sich an zwei sehr beschränkten Stellen noch Reste von Schmeckbechern, jedoch gelang es nur bei einer genauen Betrachtung des Präparates, dieselben zu entdecken, da ihre Veränderung derart war, dass nur die Anordnung der Zellen andeutete, es haben hier Schmeckbecher einst existirt. Die Spalten zwischen den Falten, in welchen die Reste der Schmeckbecher vorkommen, zeigten sehr ausgeprägt jene Veränderung, von der wir bald sprechen werden. 
In der Papilla vallata desselben Kaninchens, von welcher 6 brauchbare Schnitte gewonnen wurden, fehlten in vier derselben, sowohl im Epithel des Walles, als auch am seitlichen Abtange der Papille, die Schmeckbecher gänzlich; an den zwei anderen Präparaten liess sich noch die Gegenwart dieser Gebilde, wenn auch nicht sehr deutlich erkennen, und zwar waren in einem Präparate zwei, in dem anderen blos ein Schmeckbecher im Epithel am Seitenabhang der Papilla vorhanden.

Die Präparate aus der Papilla foliaca des I. Kaninchens lieferten uns im Allgemeinen dasselbe Resultat, als jene desselben Organs vom II. Kaninchen. Wir dürfen jedoch nicht verschweigen, dass auch bei diesem Kaninchen an zwei Schnitten der Pap. foliata sehr spärliche Schmeckbecher vorkamen.

Die Scbuitte treffen die ganze Pap. foliata, so dass es möglich ist, alle Schleimhautfalten zu sehen. In einem Schnitt fanden wir blos einen, in dem andern drei oder vier über einander gelegene Schmeckbecher, deren Contouren aber nicht scharf markirt waren. Alle uibrigen Falten enthielten gar keine Schmeckbecher und die Anordnung der Epithelzellen liess nicht einmal errathen, dass je vorher eine Geschmacksknospe vorhanden gewesen sei.

In der Pap. vallata dieses I. Kaninchens waren gar keine Schmeckbecher, weder im Epithel des Walles, noch am Seitenabhang der Papille, vorhanden.

Nachdem nun an beiden Kaninchen auf der nicht operirten Seite die Schmeckbecher wohl ausgebildet vorkommen, so müssen wir schliessen, dass gewiss nach Ablauf von fünf Monaten nach Durchschneidung des N. glossopharyngeus die Schmeckbecher verschwinden und an ihrer Stelle bloss gewöhnliche Epithelzellen auftreten.

Bei Untersuchung der Präparate aus der Pap. fol. des II. Kaninchens fiel uns noch eine andere Erscheinung auf.

Wenn man die Schnitte bei einer sehr schwachen Vergrösserung untersucht, bei einer solchen nämlich, bei welcher die Schmeckbecher nicht unterschieden werden können, so sieht man, dass auf der gesunden Seite die Spalten zwischen den einzelnen Geschmacksleisten viel tiefer nach abwärts reichen als auf der operirten Seite, wo bloss die Firste derselben getrennt erscheinen. Aus dieser Erscheinung allein konnten wir entscheiden, ob der vorgelegte Schnitt aus der gesunden, oder aus der operirten Seite entnommen wurde. 
Beim I. Kaninchen war dagegen die angeführte Erscheinung so wenig ausgeprägt, dass bei einer schwachen Vergrösserung die eben angedeutete Unterscheidung nicht möglich war.

Da aber die Präparate behufs der Bereitung der Schnitte eingebettet wurden, so könnte man leicht daran denken, dass wir es beim II. Kaninchen bloss mit einer zufälligen Erscheinung zu thun hätten; nämlich, mit einer zufälligen Zusammenpressung der Falten durch die Einbettungsmasse; wir untersuchten deshalb die Präparate auch mit starken Vergrösserungen.

Diese Untersuchung ergab, dass beim II. Kaninchen das Seitenepithel einer Falte mit dem Seitenepithel der nächststehenden Falte in den meisten Fällen so innig zusammenhängt, dass zwischen beiden keine Andeutung einer Trennung vorkommt; in anderen Fällen sind die Epithelzellen in der Mitte der ehemaligen Spalte etwas lockerer, jedoch auch in diesen Fällen kann man von einer eigentlichen Spalte nicht reden. Nur an den oberftächlichsten Partieen der Falten sieht man deutlich, wie dieselben von einander getrennt sind.

Beim Kaninchen I ist diese Erscheinung weniger ausgeprägt; nämlich blos in der Tiefe der Spalte ist das Epithel einer Falte mit jenem der benachbarten in inniger Berührung; es sieht so aus, als ob bei diesem I. Kaninchen die Veränderung nicht so weit vorgeschritten sei, wie beim II.

Wir bemerken hier ausdrücklich, dass, wie bekannt, der Grund einer Spalte auch bei einer gesunden Pap. foliata mit Epithelzellen ausgekleidet ist; bei einer Pap. foliata aber, in welcher die Schmeckbecher in Folge der vor Monaten ausgeführten Durchschneidung des Glossopharyngeus fehlen, reicht dieses Epithel bedeutend höher hinauf, und man kann leicht erkennen, dass die oberflächlichen Zellen mit ihrem langen Durchmesser parallel der Falte liegen.

Man gewinnt nach der Betrachtung solcher Präparate die Ueberzeugung, als ob nach Durchschneidung des Glossopharyngeus nicht blos die Schmeekbecher verschwinden, sondern dass auch das Bestreben vorhanden sei, die Spalten, deren physiologische Nothwendigkeit nicht mehr existirt, mit Epithel auszufüllen, dieselben $\mathrm{zu}$ verwischen.

Eine Erklärung, warum beim I. Kaninchen die Furchen zwischen den Falten weniger mit Epithel ausgefüllt waren als beim II. Kaninchen, können wir nicht anführen, und wir sprechen blos die Vermuthung 
aus, dass das II. Kaninchen vielleicht jünger war als das I., da beide nicht aus demselben Wurfe stammten.

Dass die beobachteten Veränderungen nicht etwa von der alleinigen Bloslegung des Nerven abhängen, lässt sich daraus schliessen, dass bei einem dritten Kaninchen alle die operativen Eingriffe mit Ausnahme der Durchschneidung des Glossopharyngeus zur selben Zeit wie bei den zwei anderen vorgenommen wurden, und weder die Schmeckbecher noch die Spalten zwischen den einzelnen Falten irgend eine Veränderung zeigten. Die oben besprochenen Versuche liefern den sichersten Beweis, dass die Schmeckbecher mit dem Glossopharyngeus in innigster Verbindung stehen.

Es ist nun Sache der mikroskopischen Forschung, über die Art dieser Verbindung endgültig zu endscheiden, wie auch zu ermitteln, welche Veränderungen die Schmeckbecher durchmachen, bis dieselben vollständlg verschwinden, und an ihrer Stelle nur Platten - Epithelzellen auftreten.

Die Beantwortung der Frage, ob die Schmeckbecher in Folge der Durchschneidung der Nerven, welche zu denselben gehen, eine Veränderung erleiden, war für uns noch in anderer Hinsicht von Bedeutung; denn es ist vielleicht möglich, auf diesem Weg die Frage zu entscheiden, welche Nerven den Geschmack an der Zungenspitze und den Zungenrändern vermitteln.

Auf die verschiedenen Fragen, welche nun durch unsere Beobachtungen entstehen, werden wir später eingehen.

\section{Berichtigende Bemerkung zu G. Colasanti's Beitrag zur Fieberlehre.}

(S. dies. Archiv Bd. XIV. S. 125.)

Von

Prof. Dr. H. Senator.

Bei Gelegenheit seiner Versuche über den Einfluss der verschiedenen Umgebungstemperaturen auf die Grössen des Gaswechsels von Meerschweinchen hat Colasanti auch eine Beobachtung an einem fiebernden Thiere gemacht und als Beitrag zur Fieber- 